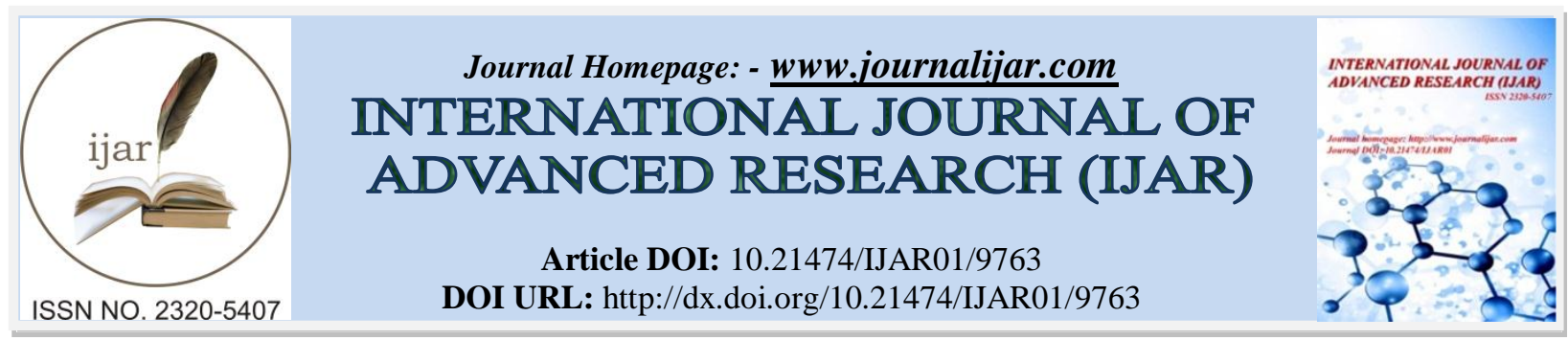

RESEARCH ARTICLE

\title{
A COMPARATIVE EVALUATION OF THE EFFECT OF PANCHMULABALADI KSHEER BASTI \& RASNADI GUGGULU IN THE MANAGEMENT OF SANDHIVATA.
}

Aparna Tripathi and S.K. Tiwari.

\section{Manuscript Info}

\section{Manuscript History}

Received: 12 July 2019

Final Accepted: 14 August 2019

Published: September 2019

Key words:-

Sandhivata, Osteoarthritis, Rasnadi guggulu, Panchmulabaladi ksheerbasti, kala basti.

\begin{abstract}
Ayurveda defines body as something that is constantly going through the process of decay- shiryate iti shariram ${ }^{1}$, here decay indicates gradual ageing of the body. Ageing is a complex process of accumulation of molecular, cellular, and organ damage, leading to loss of function and increased vulnerability to disease and death, the rate and extent of which varies among individuals ${ }^{2}$. Osteoarthritis (OA) is not only the most common joint disease, but is also one of the major causes of disability in people aged $>65$ years and is accompanied by co-morbid conditions, increased mortality, and decreased quality of life. One of the major risk factors for $\mathrm{OA}$ is ageing ${ }^{3}$. With the process of ageing all dhatus undergo kshaya, and leads to vataprakopa and the vitiated Vata dosha get lodged in Asthi sandhis and give rise to Sandhigata vata. The present clinical work is based on the study of 45 patients of Sandhigatavata (Osteo-arthritis) divided into three Groups A, B and C in terms of the nature of given treatment. Group A patients were prescribed Shamana therapy with Rasnadi guggulu, group B patient were administered Panchamulabaladi ksheer basti in kalabasti schedule of 16 days, group C patients were given Rasnadi guggulu after completion of Panchmulabaladi ksheer basti as per the schedule in group B. Subjective parameters and objective parameters were made out to assess the Clinical response. All group shows statistically highly significant relief in the subjective and objective parameters.
\end{abstract}

Copy Right, IJAR, 2019,. All rights reserved.

\section{Introduction:-}

Sandhigatavata is described under Vatavyadhi in all the Samhita and Sangraha Grantha. Sandhigatavata is a Dhatukshayajanya Vyadhi, and occurs usually after mid life stage. Vardhakya is predominated by Vata dosha $^{4}$ and characterized by Dhatu kshaya ${ }^{5}$. Vataprakopaka ahara vihara along with age related dhatukshaya reduces sneha bhava and kapha in the body and allows the vitiated vata to lodge in asthivaha and majjavaha srotas ${ }^{6}$ mainly in Asthi and Sandhi thereby resulting in karmahani (loss of function) of the Sandhis. When aggravated Vata gets localized in Sandhi, it over powers and undoes all qualities of kapha .Shleshaka Kapha is described to be situated in the Sandhi ${ }^{7}$. It keeps the joints firmly united and helps in their function. Also, dhatushaithilya is another feature in Vardhakya $^{8}$, which reflects in peshis and snayus thereby reducing their functional efficiency in supporting the joints. Asthi- Sandhi is one of the component of Madhyamarogamarga ${ }^{9}$. The disease which is located in Marma and Sandhi

Corresponding Author:-Aparna Tripathi. 
is considered as Yapya ${ }^{10}$. Osteoarthritis is the most common type of arthritis in both developed and developing countries. It is a chronic degenerative disorder of multifactorial etiology characterised by the loss of articular cartilage, hypertrophy of bone at the margins, subchondral sclerosis and range of biochemical and morphological alterations of the synovial membrane and joint capsule ${ }^{11}$. It is a major cause of disability in older adults worldwide. According to World Health Organization (WHO) $9.6 \%$ of men and $18 \%$ of women aged over 60 years has symptomatic OA worldwide. $80 \%$ of those with OA have limitations in movement and $25 \%$ cannot perform their major daily activities of life ${ }^{12}$. Osteoarthritis is the second most common rheumatologic problem and it is the most frequent joint disease with a prevalence of $22 \%$ to $39 \%$ in India ${ }^{13}$.

Goal of managing Osteoarthritis includes controlling pain, maintaining and improving range of movement and stability of affected joints and limiting functional impairment ${ }^{14}$. Several pharmacological and non-pharmacological mode of treatment are in use now a days, but the toxicity and adverse event profile of the most commonly used existing treatment in contemporary medical science (such as NSAIDs, and total joint replacement) is unfavourable ${ }^{15}$. Modalities of treatment have been tried in various systems of medicine including the contemporary system of medicine but the statistical figure of the cured is still lying low than the expectant and has kept whole medical fraternity on tenterhooks.

An indigenous compound drug for shaman chikitsa, Rasnadi Guggulu ${ }^{16}$, mainly containing vatahara and rasayana drugs like Rasna, Devdaru, Erand, Guduchi, Guggulu etc was given in one group. In the another group Basti was given with Panchmulabalasiddha ksheera ${ }^{17,}{ }^{18}$. Panchamulabaladi ksheera as well as its route of administrationBasti both have vatashamaka properties. Patients of group C were given Panchmulabaladi Ksheer Basti in kalabasti ${ }^{19}$ schedule of 16 days, followed by Rasnadi guggulu as given in group A.

\section{Aims And Objectives:-}

1. To assess the effect of Rasnadi Guggulu in Sandhigatavata.

2. To evaluate the effect of Panchamulabaladi Ksheer Basti on Sandhigatavata.

3. To evaluate the additive effect of Rasnadi guggulu and Panchmulabaladi Ksheer Basti

4. To compare the difference of results in the above treatment groups.

\section{Material and methods:-}

\section{Drugs}

Table 1:-Ingredients of Rasnadi Guggulu

\begin{tabular}{|l|l|l|}
\hline S.No & Drugs & Botanical name \\
\hline 1. & Rasna & Pluchea lanceolata \\
\hline 2. & Guduchi & Tinospora cordifolia \\
\hline 3. & Eranda mula & Ricinus communis \\
\hline 4. & Deodaru & Cedrus deodara \\
\hline 5. & Shunthi & Zingiber officinale \\
\hline 6. & Guggulu & Commiphora wightii \\
\hline
\end{tabular}

Table 2:-Ingredients of Panchmulabaladi ksheer basti.

\begin{tabular}{|l|l|l|}
\hline S.No & Drugs & Botanical name \\
\hline 1. & Bilwa & Aegle marmelos \\
\hline 2. & Agnimanth & Premna mucronata \\
\hline 3. & Shyonaka & Oroxylum indicum \\
\hline 4. & Gambhari & Gmelina arborea \\
\hline 5. & Patala & Stereospermum suaveolens \\
\hline 6. & Bala & Sida cordifolia \\
\hline 7. & Saunfa & Anethum sowa \\
\hline 8. & Godugdha (milk) & - \\
\hline 9. & Madhu (Honey) & - \\
\hline 10. & Saindhava lavana (rock salt) & - \\
\hline
\end{tabular}




\section{Selection of patients:}

A series of 45 patients of Sandhivata vis-a-vis Osteoarthritis were randomly selected for the present study from OPD and IPD of Kayachikitsa, Sir Sundarlal Hospital, Institute of Medical Sciences, B.H.U, Varanasi. The cases were selected randomly on the basis of classical sign and symptoms described in various ayurvedic texts giving special importance to cardinal symptoms like Sandhishula ${ }^{20}$, Sandhigati asamarthya ${ }^{21}$, Sandhishotha ${ }^{22}$, Atopa ${ }^{23}$, Akunchana prasaranjanya vedana ${ }^{24}$ irrespective of their sex, religion and socioeconomic considerations. Out of 45 patients only 35 cases could complete their follow up i.e. 3 months. The following exclusion and inclusion criteria were adopted for the selection of cases of Osteoarthritis -

\section{Exclusion criteria:}

1. History of active presence of other inflammatory or rheumatic disease.

2. Patients below 40 years and aged above 80 years.

3. History of traumatic arthritis.

4. Over weight defined by Body mass index BMI of $>30$.

5. Substantial abnormalities in haematological, hepatic, renal or metabolic function.

\section{Inclusion criteria:}

1. Patients diagnosed as Sandhigatavata on the basis of sign and symptoms according to classical ayurvedic texts.

2. Patient fit for Basti karma.

3. Patients with radiological findings of Osteoarthritis along with clinical features.

4. Patient aged over 40 years with involvement of knee joint.

\section{GROUPING:}

After selection of the patients they were randomly divided into following groups

Group - A :

The patients of this group were administered Rasnadi Guggulu twice daily in the morning and in evening on empty stomach. In this group 15 patients were registered, out of them 14 patients completed the course.

Dose -

$50 \mathrm{mg} / \mathrm{kg}$ body weight in divided doses.

\section{Anupana -}

Luke worm water, patients were suggested to take drug after crushing the bati.

\section{Group - B}

In this group, Panchmulabaladi Ksheer Basti in Kala basti schedule of 16 days was administered. Patients were advised Panchkola churna 3gm bid after meal for 3-5 days for Amapachana. Abhyanga with Bala tail and mridu nadi swedana was done prior to basti treatment as purvakarma. In this group 15 patients were registered, out of which 10 patients completed their course.

Dose :-

Bala tail Anuvasan Basti - 80 ml. Panchmulabaladi Ksheer Basti- 450-500 ml.

\section{Group - C}

Patients of this group were given Rasndi guggulu after completion of Panchmulabaladi Ksheer Basti as per the schedule of group A and B.

In this group 15 patents were registered out of them 11 patients completed the course.

\section{Assessment Of Clinical Response}

Subjective parameters and objective parameters were made out to assess the clinical response in all the groups. 


\section{Subjective Parameters}

Prasarana-akunchanayo-savedana (pain)

\begin{tabular}{|l|l|l|}
\hline 01. & Grade 0 & No Complaints \\
\hline 02 & Grade 1 & Tells on Enquiry \\
\hline 03 & Grade 2 & Complains Frequently \\
\hline 04 & Grade 3 & Excruciating Condition \\
\hline
\end{tabular}

Stambha (Stiffness)

\begin{tabular}{|l|l|l|}
\hline 01. & Grade 0 & Absent \\
\hline 02. & Grade 1 & Present \\
\hline
\end{tabular}

\section{Objective parameters}

Sparshaakshamatva (Tenderness)

\begin{tabular}{|l|l|l|}
\hline 01. & Grade 0 & No Complaints \\
\hline 02. & Grade 1 & Says the joint is tender \\
\hline 03. & Grade 2 & Winces the affected joint \\
\hline 04. & Grade 3 & Winces and withdraws the affected joint. \\
\hline
\end{tabular}

Atopa (Crepitations)

\begin{tabular}{|l|l|l|}
\hline 01. & Grade 0 & None \\
\hline 02. & Grade 1 & Felt \\
\hline 03. & Grade 2 & Heard \\
\hline
\end{tabular}

Sandhigati-Asaamarthya (Restriction of joint movement)

\begin{tabular}{|l|l|l|}
\hline 01. & Grade 4 & No movement \\
\hline 02. & Grade 3 & Up to $50 \%$ of the full range of joint motion. \\
\hline 03. & Grade 2 & $50-75 \%$ of the full range of joint motion \\
\hline 04. & Grade 1 & $>75 \%$ \& < full range \\
\hline
\end{tabular}

Shotha (Swelling)

\begin{tabular}{|l|l|l|}
\hline 01. & Grade 0 & No Complaints \\
\hline 02. & Grade 1 & Slightly obvious \\
\hline 03. & Grade 2 & Covers well over the bony prominence \\
\hline 04. & Grade 3 & Much elevated \\
\hline
\end{tabular}

Walking time to cover 21 meters distance:

\begin{tabular}{|l|l|l|}
\hline 01. & Grade 0 & Up to 20seconds \\
\hline 02. & Grade 1 & $21-30$ seconds \\
\hline 03. & Grade 2 & $31-40$ seconds \\
\hline 04. & Grade 3 & $41-50$ seconds \\
\hline 05. & Grade 4 & $51-60$ seconds \\
\hline
\end{tabular}

Overall Assessment of Clinical Response:

\begin{tabular}{|l|l|l|}
\hline 1. & Good Response & $>75 \%$ improvement in overall clinical parameters. \\
\hline 2. & Moderate Response & $50-75 \%$ improvement in overall clinical parameters. \\
\hline 3. & Poor Response & Up to $50 \%$ improvement in overall clinical parameters \\
\hline 4. & No Response & $0 \%$ or No improvement in overall clinical parameters \\
\hline
\end{tabular}

\section{Womac Scale}

The Western Ontario and McMaster Osteoarthritis Index (WOMAC) is a disease specific, Self-administered, health status measure of symptoms and physical disability. The WOMAC is considered the leading outcome measure for 
patients with OA of the lower extremities. Numerous studies have reported on its reliability and validity. There have also been several studies, which have raised issues about the factorial validity of the subscales.

The WOMAC has three subscales Pain -5 questions, Stiffness -2 questions, Physical functioning -17 questions $^{25}$. Each question uses a likert scale from 0-4 i.e:

$\begin{array}{llll}\text { 1. None } & : & 0 \\ \text { 2. } & \text { Mild } & : & 1 \\ \text { 3. } & \text { Moderate } & : & 2 \\ \text { 4. Sever } & : & 3 \\ \text { 5. } & \text { Extreme } & : & 4\end{array}$

Interpretation:

Higher scores on WOMAC indicate worse pain, stiffness and functional limitations.

1. Minimum total score: 0

2. Maximum total score: 96

\section{Pain subscale items}

1. Walking on the flat surface

2. Going up or down stairs

3. At night while in bed

4. Sitting or lying

5. Standing upright.

\section{Stiffness scale items:}

How severe is your knee joint stiffness after first wakening in the morning and later in the day.

\section{Physical functioning subscale:}

1. Descending stairs

2. Ascending stairs

3. Rising from sitting

4. Standing

5. Bending to floor

6. Walking on flat

7. Getting in or out of car

8. Going shopping

9. Putting on socks

10. Rising from bed

11. Taking off socks

12. Lying in bed

13. Sitting

14. In/out bath

15. Getting on or off toilet

16. Heavy domestic duties

17. Light domestic duties

\section{Statistical analysis:}

For analysis of pain, stiffness, tenderness, swelling, restriction of movement, crepitus and walking time, within the group comparison was done by friedman's Chi-Square test.

Between the groups comparison was done by PEARSON's Chi- Square test. Wherever expected frequency came less than 5, Chi-Square has been calculated after pulling the suitable rows or columns. For WOMAC score, within the group comparison was done by Paired t' test and calculating the mean difference of before and after the treatment. 
Between the groups comparison was done by One Way ANOVA test. Post-Hoc test was done to compare significant pair of groups.

\section{Statistical Significance}

1. $\mathrm{p}<0.05$ was considered statistically significant and

2. $\mathrm{p}<0.01$ or $\mathrm{p}<0.001$ as statistically highly significant

3. $\mathrm{p}>0.05$ as not statistically significant

\section{Observations and Results:-}

\section{Observation of demographic data:}

Table 4:-Age wise distribution of 45 Patients of Sandhigatavata

\begin{tabular}{|c|c|c|c|}
\hline \multirow{2}{*}{ Sr. } & Age (year ) & \multicolumn{2}{|c|}{ Total } \\
\cline { 3 - 4 } & & Number & Percentage \\
\hline 1. & $30-40$ & 5 & $11.11 \%$ \\
\hline 2. & $41-50$ & 21 & $46.66 \%$ \\
\hline 3. & $51-60$ & 12 & $26.66 \%$ \\
\hline 4. & $61-70$ & 7 & $15.55 \%$ \\
\hline
\end{tabular}

Table 5:-Sex wise distribution of 45 Patients of Sandhigatavata

\begin{tabular}{|l|l|c|c|}
\hline \multirow{2}{*}{ Sr. } & \multicolumn{1}{|c|}{ Sex } & \multicolumn{2}{|c|}{ Total } \\
\cline { 3 - 4 } & & Number & Percentage \\
\hline 1. & Male & 17 & $37.77 \%$ \\
\hline 2. & Female & 28 & $62.22 \%$ \\
\hline
\end{tabular}

Table 6:-Occupation wise distribution of 45 patients of Sandhi- gatavata

\begin{tabular}{|l|l|c|c|}
\hline \multirow{2}{*}{ Occupation } & \multicolumn{2}{c|}{ Total } \\
\cline { 3 - 4 } & & Number & Percentage \\
\hline 1. & Sedentary & 14 & $31.11 \%$ \\
\hline 2. & Active & 25 & $55.55 \%$ \\
\hline 3. & Labour & 6 & $13.33 \%$ \\
\hline 4. & Other & 0 & $0 \%$ \\
\hline
\end{tabular}

Table 7:-Pattern of Onset wise distribution of 45 Patients of Sandhigatavata

\begin{tabular}{|c|l|c|c|}
\hline \multirow{2}{*}{ Sr. } & \multicolumn{1}{|c|}{ Pattern of onset } & \multicolumn{2}{|c|}{ Total } \\
\cline { 3 - 4 } & & Number & Percentage \\
\hline 1. & Gradual & 36 & $80 \%$ \\
\hline 2. & Insidious & 9 & $20 \%$ \\
\hline
\end{tabular}

Table 8:-Chronicity wise distribution of 45 Patients of Sandhigatavata

\begin{tabular}{|c|l|c|c|}
\hline \multirow{2}{*}{ Sr. Chronicity } & \multicolumn{2}{|c|}{ Total } \\
\cline { 3 - 4 } & & Number & Percentage \\
\hline 1. & $0-6 \mathrm{mnths}$ & 5 & $11.11 \%$ \\
\hline 2. & $6-12 \mathrm{mnths}$ & 7 & $15.55 \%$ \\
\hline 3. & $1-2 \mathrm{yrs}$ & 15 & $33.33 \%$ \\
\hline 4. & $2-5 \mathrm{yrs}$ & 8 & $17.77 \%$ \\
\hline 5. & $>5 \mathrm{yrs}$ & 10 & $22.22 \%$ \\
\hline
\end{tabular}

Table 9:-Kostha wise distribution of 45 Patients of Sandhigatavata

\begin{tabular}{|c|c|c|c|}
\hline Sr. & \multicolumn{1}{|c|}{ Kostha } & \multicolumn{2}{|c|}{ Total } \\
\cline { 3 - 4 } & & Number & Percentage \\
\hline 1. & Mridu & 6 & $13.33 \%$ \\
\hline
\end{tabular}




\begin{tabular}{|l|l|c|c|}
\hline 2. & Madhya & 15 & $33.33 \%$ \\
\hline 3. & Krura & 24 & $53.33 \%$ \\
\hline
\end{tabular}

Data Related to clinical features before treatment and after follow-up:

Subjective parametres :

Table 10:-Showing the distribution of patients by different grades of Vedana .

Prasaranaakunchanayohosavedanapravruthihi:( Pain on movement )

\begin{tabular}{|c|c|c|c|c|c|c|}
\hline \multirow[t]{2}{*}{ Group } & \multirow[t]{2}{*}{ Grade } & \multicolumn{4}{|c|}{ No. of cases with percentage } & \multirow{2}{*}{$\begin{array}{c}\text { Within the } \\
\text { group } \\
\text { comparison } \\
\text { Friedman Test }\end{array}$} \\
\hline & & BT & $\mathrm{F} 1$ & $\mathrm{~F} 2$ & F3 & \\
\hline \multirow{4}{*}{$\begin{array}{l}\text { Group A } \\
(n=14)\end{array}$} & 0 & $\begin{array}{c}0 \\
0 \% \\
\end{array}$ & $\begin{array}{c}0 \\
0 \%\end{array}$ & $\begin{array}{c}0 \\
0 \%\end{array}$ & $\begin{array}{c}2 \\
14.3 \% \\
\end{array}$ & \multirow{4}{*}{$\begin{array}{l}\chi^{2}=27.600 \\
P<.001\end{array}$} \\
\hline & 1 & $\begin{array}{c}0 \\
0 \%\end{array}$ & $\begin{array}{c}1 \\
7.1 \%\end{array}$ & $\begin{array}{c}3 \\
21.4 \%\end{array}$ & $\begin{array}{c}4 \\
28.6 \%\end{array}$ & \\
\hline & 2 & $\begin{array}{c}6 \\
42.9 \%\end{array}$ & $\begin{array}{c}7 \\
50 \%\end{array}$ & $\begin{array}{c}10 \\
71.4 \%\end{array}$ & $\begin{array}{c}8 \\
57.1 \%\end{array}$ & \\
\hline & 3 & $\begin{array}{c}8 \\
57.1 \%\end{array}$ & $\begin{array}{c}6 \\
42.9 \%\end{array}$ & $\begin{array}{c}1 \\
7.1 \%\end{array}$ & $\begin{array}{c}0 \\
0 \%\end{array}$ & \\
\hline \multirow{4}{*}{$\begin{array}{l}\text { Group B } \\
(\mathrm{n}=10)\end{array}$} & 0 & $\begin{array}{c}0 \\
0 \% \\
\end{array}$ & $\begin{array}{c}0 \\
0 \% \\
\end{array}$ & $\begin{array}{c}0 \\
0 \% \\
\end{array}$ & $\begin{array}{c}2 \\
20 \% \\
\end{array}$ & \multirow{4}{*}{$\begin{array}{l}\chi^{2}=21.808 \\
P<.001\end{array}$} \\
\hline & 1 & $\begin{array}{c}0 \\
0 \%\end{array}$ & $\begin{array}{c}6 \\
60 \%\end{array}$ & $\begin{array}{c}7 \\
70 \%\end{array}$ & $\begin{array}{c}7 \\
70 \%\end{array}$ & \\
\hline & 2 & $\begin{array}{c}4 \\
40 \% \\
\end{array}$ & $\begin{array}{c}2 \\
20 \%\end{array}$ & $\begin{array}{c}3 \\
30 \% \\
\end{array}$ & $\begin{array}{c}1 \\
10 \% \\
\end{array}$ & \\
\hline & 3 & $\begin{array}{c}6 \\
60 \%\end{array}$ & $\begin{array}{c}2 \\
20 \%\end{array}$ & $\begin{array}{c}0 \\
0 \%\end{array}$ & $\begin{array}{c}0 \\
0 \%\end{array}$ & \\
\hline \multirow{4}{*}{$\begin{array}{l}\text { Group C } \\
(n=11)\end{array}$} & 0 & $\begin{array}{c}0 \\
0 \%\end{array}$ & $\begin{array}{c}0 \\
0 \%\end{array}$ & $\begin{array}{c}0 \\
0 \%\end{array}$ & $\begin{array}{c}5 \\
45.5 \%\end{array}$ & \multirow{4}{*}{$\begin{array}{l}\chi^{2}=23.080 \\
P<.001\end{array}$} \\
\hline & 1 & $\begin{array}{c}0 \\
0 \%\end{array}$ & $\begin{array}{c}5 \\
45.5 \%\end{array}$ & $\begin{array}{c}6 \\
54.5 \%\end{array}$ & $\begin{array}{c}3 \\
27.3 \%\end{array}$ & \\
\hline & 2 & $\begin{array}{c}4 \\
36.4 \%\end{array}$ & $\begin{array}{c}3 \\
27.3 \%\end{array}$ & $\begin{array}{c}4 \\
36.4 \%\end{array}$ & $\begin{array}{c}2 \\
18.2 \%\end{array}$ & \\
\hline & 3 & $\begin{array}{c}7 \\
63.6 \%\end{array}$ & $\begin{array}{c}3 \\
27.3 \%\end{array}$ & $\begin{array}{c}1 \\
9.1 \%\end{array}$ & $\begin{array}{c}1 \\
9.1 \%\end{array}$ & \\
\hline $\begin{array}{l}\text { Between the } \\
\text { Group } \\
\text { comparison }\end{array}$ & $\begin{array}{l}\text { Pearson Chi- } \\
\text { Square Test }\end{array}$ & $\begin{array}{l}\chi^{2}=0.11 \\
P>.05\end{array}$ & $\begin{array}{l}\chi^{2}=1.54 \\
P>.05\end{array}$ & $\begin{array}{l}\chi^{2}=0.98 \\
P>.05\end{array}$ & $\begin{array}{l}\chi^{2}=6.10 \\
P<.05\end{array}$ & \\
\hline
\end{tabular}

Table 11 :-Stambha ( stiffness )

\begin{tabular}{|c|c|c|c|c|c|c|}
\hline \multirow[t]{2}{*}{ Group } & \multirow[t]{2}{*}{ Grade } & \multicolumn{4}{|c|}{ No. of cases with percentage } & \multirow{2}{*}{$\begin{array}{l}\text { Within the group } \\
\text { comparison } \\
\text { Friedman Test }\end{array}$} \\
\hline & & BT & F1 & F2 & F3 & \\
\hline \multirow[t]{2}{*}{ Group A } & $\mathbf{0}$ & $\begin{array}{c}2 \\
14.3 \%\end{array}$ & $\begin{array}{c}3 \\
21.4 \%\end{array}$ & $\begin{array}{c}3 \\
21.4 \%\end{array}$ & $\begin{array}{c}5 \\
35.7 \%\end{array}$ & \multirow{2}{*}{$\begin{array}{l}\chi^{2}=6.333 \\
p>.05\end{array}$} \\
\hline & 1 & $\begin{array}{c}12 \\
85.7 \%\end{array}$ & $\begin{array}{c}11 \\
78.6 \%\end{array}$ & $\begin{array}{c}11 \\
21.4 \%\end{array}$ & $\begin{array}{c}9 \\
64.3 \%\end{array}$ & \\
\hline \multirow{3}{*}{ Group B } & $\mathbf{0}$ & $\begin{array}{c}1 \\
10 \%\end{array}$ & $\begin{array}{c}5 \\
50 \%\end{array}$ & $\begin{array}{c}6 \\
60 \%\end{array}$ & $\begin{array}{c}7 \\
70 \% \\
\end{array}$ & \multirow{2}{*}{$\begin{array}{l}\chi^{2}=13.105 \\
p<.01\end{array}$} \\
\hline & 1 & $\begin{array}{c}9 \\
90 \% \\
\end{array}$ & $50 \%$ & $\begin{array}{c}4 \\
40 \% \\
\end{array}$ & $\begin{array}{c}3 \\
30 \% \\
\end{array}$ & \\
\hline & $\mathbf{0}$ & 1 & 6 & 6 & 9 & $\chi^{2}=16.500$ \\
\hline
\end{tabular}




\begin{tabular}{|c|c|c|c|c|c|c|}
\hline \multirow[b]{2}{*}{ Group C } & & $9.1 \%$ & $54.5 \%$ & $54.5 \%$ & $81.8 \%$ & \multirow[t]{2}{*}{$\mathrm{p}<.001$} \\
\hline & 1 & $\begin{array}{c}10 \\
90.9 \%\end{array}$ & $\begin{array}{c}5 \\
45.5 \%\end{array}$ & $\begin{array}{c}5 \\
45.5 \%\end{array}$ & $\begin{array}{c}2 \\
18.2 \%\end{array}$ & \\
\hline $\begin{array}{l}\text { Between the } \\
\text { Group } \\
\text { comparison }\end{array}$ & $\begin{array}{l}\text { PEARSON } \\
\text { Chi-Square } \\
\text { Test }\end{array}$ & $\begin{array}{l}\chi^{2}=0.19 \\
p>.05\end{array}$ & $\begin{array}{l}\chi^{2}=3.40 \\
p>.05\end{array}$ & $\begin{array}{l}\chi^{2}=4.44 \\
p>.05\end{array}$ & $\begin{array}{l}\chi^{2}=6.04 \\
p<.05\end{array}$ & \\
\hline
\end{tabular}

Objective Parameters

Table 12:-Sparsha akshamatva (tenderness)

\begin{tabular}{|c|c|c|c|c|c|c|}
\hline \multirow[t]{2}{*}{ Group } & \multirow[t]{2}{*}{ Grade } & \multicolumn{4}{|c|}{ No. of cases with percentage } & \multirow{2}{*}{$\begin{array}{c}\text { Within the } \\
\text { group } \\
\text { comparison } \\
\text { Friedman Test }\end{array}$} \\
\hline & & BT & F1 & F2 & $\mathbf{F 3}$ & \\
\hline \multirow{4}{*}{$\begin{array}{l}\text { Group A } \\
(n=14)\end{array}$} & $\mathbf{0}$ & $\begin{array}{c}2 \\
14.3 \%\end{array}$ & $\begin{array}{c}4 \\
28.6 \%\end{array}$ & $\begin{array}{c}4 \\
28.6 \%\end{array}$ & $\begin{array}{c}5 \\
35.7 \%\end{array}$ & \multirow{4}{*}{$\begin{array}{l}\chi^{2}=22.726 \\
P<.001\end{array}$} \\
\hline & 1 & $\begin{array}{c}2 \\
14.3 \%\end{array}$ & $\begin{array}{c}3 \\
21.4 \%\end{array}$ & $\begin{array}{c}4 \\
28.6 \%\end{array}$ & $\begin{array}{c}5 \\
35.7 \%\end{array}$ & \\
\hline & 2 & $\begin{array}{c}7 \\
50 \% \\
\end{array}$ & $\begin{array}{c}5 \\
35.7 \%\end{array}$ & $\begin{array}{c}5 \\
35.7 \% \\
\end{array}$ & $\begin{array}{c}4 \\
28.6 \% \\
\end{array}$ & \\
\hline & 3 & $\begin{array}{c}3 \\
21.4 \% \\
\end{array}$ & $\begin{array}{c}2 \\
14.3 \%\end{array}$ & $\begin{array}{c}1 \\
7.1 \% \\
\end{array}$ & $\begin{array}{l}0 \\
0 \%\end{array}$ & \\
\hline \multirow{4}{*}{$\begin{array}{l}\text { Group B } \\
(n=10)\end{array}$} & $\mathbf{0}$ & $\begin{array}{l}0 \\
0 \%\end{array}$ & $\begin{array}{c}1 \\
10 \%\end{array}$ & $\begin{array}{c}1 \\
10 \%\end{array}$ & $\begin{array}{c}5 \\
50 \%\end{array}$ & \multirow{4}{*}{$\begin{array}{l}\chi^{2}=21.507 \\
p<.001\end{array}$} \\
\hline & 1 & $\begin{array}{c}2 \\
20 \%\end{array}$ & $\begin{array}{c}3 \\
30 \%\end{array}$ & $\begin{array}{l}7 \\
70 \%\end{array}$ & $\begin{array}{c}3 \\
30 \%\end{array}$ & \\
\hline & 2 & $\begin{array}{c}3 \\
30 \%\end{array}$ & $\begin{array}{c}5 \\
50 \%\end{array}$ & $\begin{array}{c}1 \\
10 \%\end{array}$ & $\begin{array}{c}1 \\
10 \%\end{array}$ & \\
\hline & 3 & $\begin{array}{l}5 \\
50 \%\end{array}$ & $\begin{array}{c}1 \\
10 \%\end{array}$ & $\begin{array}{c}1 \\
10 \%\end{array}$ & $\begin{array}{c}1 \\
10 \%\end{array}$ & \\
\hline \multirow{4}{*}{$\begin{array}{l}\text { Group C } \\
(n=11)\end{array}$} & $\mathbf{0}$ & $\begin{array}{l}0 \\
0 \%\end{array}$ & $\begin{array}{l}0 \\
0 \%\end{array}$ & $\begin{array}{l}1 \\
9.1 \%\end{array}$ & $\begin{array}{l}7 \\
63.6 \%\end{array}$ & \multirow{4}{*}{$\begin{array}{l}\chi^{2}=24.038 \\
\mathrm{p}<.001\end{array}$} \\
\hline & 1 & $\begin{array}{l}0 \\
0 \%\end{array}$ & $\begin{array}{l}5 \\
45.5 \%\end{array}$ & $\begin{array}{c}6 \\
54.5 \%\end{array}$ & $\begin{array}{c}2 \\
18.2 \%\end{array}$ & \\
\hline & 2 & $\begin{array}{c}8 \\
72.7 \%\end{array}$ & $\begin{array}{c}5 \\
45.5 \% \\
\end{array}$ & $\begin{array}{c}3 \\
27.3 \% \\
\end{array}$ & $\begin{array}{c}1 \\
9.1 \%\end{array}$ & \\
\hline & 3 & $\begin{array}{c}3 \\
27.3 \%\end{array}$ & $\begin{array}{c}1 \\
9.1 \%\end{array}$ & $\begin{array}{l}1 \\
9.1 \%\end{array}$ & $\begin{array}{c}1 \\
9.1 \%\end{array}$ & \\
\hline $\begin{array}{l}\text { Between the } \\
\text { Group } \\
\text { comparison }\end{array}$ & $\begin{array}{l}\text { PEARSON Chi- } \\
\text { Square Test }\end{array}$ & $\begin{array}{l}\chi^{2}=3.62 \\
p>.05\end{array}$ & $\begin{array}{l}\chi^{2}=0.24 \\
p>.05\end{array}$ & $\begin{array}{l}\chi^{2}=0.502 \\
p>.05\end{array}$ & $\begin{array}{l}\chi^{2}=0.442 \\
p>.05\end{array}$ & \\
\hline
\end{tabular}

Table 13:-Shotha (swelling )

\begin{tabular}{|c|c|c|c|c|c|c|}
\hline \multirow[t]{2}{*}{ Group } & \multirow[t]{2}{*}{ Grade } & \multicolumn{4}{|c|}{ No. of cases with percentage } & \multirow{2}{*}{$\begin{array}{l}\text { Within the group } \\
\text { comparison } \\
\text { Friedman Test }\end{array}$} \\
\hline & & BT & F1 & $\mathbf{F 2}$ & F3 & \\
\hline \multirow{3}{*}{$\begin{array}{l}\text { Group A } \\
(n=14)\end{array}$} & $\mathbf{0}$ & $\begin{array}{c}1 \\
7.1 \%\end{array}$ & $\begin{array}{c}1 \\
7.1 \%\end{array}$ & $\begin{array}{c}1 \\
7.1 \%\end{array}$ & $\begin{array}{c}4 \\
28.6 \%\end{array}$ & \multirow{3}{*}{$\begin{array}{l}\chi^{2}=25.061 \\
p<.001\end{array}$} \\
\hline & 1 & $\begin{array}{c}0 \\
0 \%\end{array}$ & $\begin{array}{c}1 \\
7.1 \% \\
\end{array}$ & $\begin{array}{c}2 \\
14.3 \% \\
\end{array}$ & $\begin{array}{c}5 \\
35.7 \% \\
\end{array}$ & \\
\hline & 2 & $\begin{array}{c}7 \\
50 \%\end{array}$ & $\begin{array}{c}10 \\
71.4 \%\end{array}$ & $\begin{array}{c}11 \\
78.6 \%\end{array}$ & $\begin{array}{c}5 \\
35.7 \%\end{array}$ & \\
\hline
\end{tabular}




\begin{tabular}{|c|c|c|c|c|c|c|}
\hline & 3 & $\begin{array}{c}6 \\
42.9 \%\end{array}$ & $\begin{array}{c}2 \\
14.3 \%\end{array}$ & $\begin{array}{c}0 \\
0 \%\end{array}$ & $\begin{array}{c}0 \\
0 \%\end{array}$ & \\
\hline \multirow{4}{*}{$\begin{array}{l}\text { Group B } \\
(n=10)\end{array}$} & $\mathbf{0}$ & $\begin{array}{c}0 \\
0 \%\end{array}$ & $\begin{array}{c}2 \\
20 \%\end{array}$ & $\begin{array}{c}2 \\
20 \%\end{array}$ & $\begin{array}{c}2 \\
20 \%\end{array}$ & \multirow{4}{*}{$\begin{array}{l}\chi^{2}=20.571 \\
p<.001\end{array}$} \\
\hline & 1 & $\begin{array}{c}2 \\
20 \%\end{array}$ & $\begin{array}{c}6 \\
60 \%\end{array}$ & $\begin{array}{c}6 \\
60 \%\end{array}$ & $\begin{array}{c}6 \\
60 \%\end{array}$ & \\
\hline & 2 & $\begin{array}{c}7 \\
70 \%\end{array}$ & $\begin{array}{c}2 \\
20 \%\end{array}$ & $\begin{array}{c}2 \\
20 \%\end{array}$ & $\begin{array}{c}2 \\
20 \%\end{array}$ & \\
\hline & 3 & $\begin{array}{c}1 \\
10 \% \\
\end{array}$ & $\begin{array}{c}0 \\
0 \%\end{array}$ & $\begin{array}{c}0 \\
0 \% \\
\end{array}$ & $\begin{array}{c}0 \\
0 \% \\
\end{array}$ & \\
\hline \multirow{4}{*}{$\begin{array}{l}\text { Group C } \\
(n=11)\end{array}$} & $\mathbf{0}$ & $\begin{array}{c}0 \\
0 \%\end{array}$ & $\begin{array}{c}2 \\
18.2 \%\end{array}$ & $\begin{array}{c}2 \\
18.2 \%\end{array}$ & $\begin{array}{c}7 \\
63.6 \%\end{array}$ & \multirow{4}{*}{$\begin{array}{l}\chi^{2}=24.117 \\
p<.001\end{array}$} \\
\hline & 1 & $\begin{array}{c}2 \\
18.2 \%\end{array}$ & $\begin{array}{c}4 \\
36.4 \%\end{array}$ & $\begin{array}{c}7 \\
63.6 \%\end{array}$ & $\begin{array}{c}2 \\
18.2 \%\end{array}$ & \\
\hline & 2 & $\begin{array}{c}6 \\
54.5 \%\end{array}$ & $\begin{array}{c}5 \\
45.5 \%\end{array}$ & $\begin{array}{c}2 \\
18.2 \%\end{array}$ & $\begin{array}{c}2 \\
18.2 \% \\
\end{array}$ & \\
\hline & 3 & $\begin{array}{c}3 \\
27.3 \% \\
\end{array}$ & $\begin{array}{c}0 \\
0 \%\end{array}$ & $\begin{array}{c}0 \\
0 \%\end{array}$ & $\begin{array}{c}0 \\
0 \%\end{array}$ & \\
\hline $\begin{array}{l}\text { Between the } \\
\text { Group } \\
\text { comparison }\end{array}$ & $\begin{array}{c}\text { PEARSON } \\
\text { Chi-Square } \\
\text { Test }\end{array}$ & $\begin{array}{l}\chi^{2}=0.99 \\
p>0.05\end{array}$ & $\begin{array}{l}\chi^{2}=10.65 \\
p>.05\end{array}$ & $\begin{array}{l}\chi^{2}=8.095 \\
p<.05\end{array}$ & $\begin{array}{l}\chi^{2}=0.015 \\
p>.05\end{array}$ & \\
\hline
\end{tabular}

Table 14:-Atopa (Crepitus)

\begin{tabular}{|c|c|c|c|c|c|c|}
\hline \multirow[t]{2}{*}{ Group } & \multirow[t]{2}{*}{ Grade } & \multicolumn{4}{|c|}{ No. of cases with percentage } & \multirow{2}{*}{$\begin{array}{l}\text { Within the } \\
\text { group } \\
\text { comparison } \\
\text { Friedman Test }\end{array}$} \\
\hline & & BT & F1 & $\mathrm{F} 2$ & F3 & \\
\hline \multirow{3}{*}{$\begin{array}{l}\text { Group A } \\
(n=14)\end{array}$} & $\mathbf{0}$ & $\begin{array}{c}0 \\
0 \% \\
\end{array}$ & $\begin{array}{c}0 \\
0 \% \\
\end{array}$ & $\begin{array}{c}0 \\
0 \% \\
\end{array}$ & $\begin{array}{c}3 \\
21.4 \% \\
\end{array}$ & \multirow{3}{*}{$\begin{array}{l}\chi^{2}=11.800 \\
P<.01\end{array}$} \\
\hline & 1 & $\begin{array}{c}6 \\
42.9 \% \\
\end{array}$ & $\begin{array}{c}7 \\
50 \% \\
\end{array}$ & $\begin{array}{c}7 \\
50 \% \\
\end{array}$ & $\begin{array}{c}5 \\
35.7 \% \\
\end{array}$ & \\
\hline & 2 & $\begin{array}{c}8 \\
57.1 \% \\
\end{array}$ & $\begin{array}{c}7 \\
50 \% \\
\end{array}$ & $\begin{array}{c}7 \\
50 \% \\
\end{array}$ & $\begin{array}{c}6 \\
42.9 \% \\
\end{array}$ & \\
\hline \multirow{3}{*}{$\begin{array}{l}\text { Group B } \\
(n=10)\end{array}$} & $\mathbf{0}$ & $\begin{array}{c}0 \\
0 \% \\
\end{array}$ & $\begin{array}{c}2 \\
20 \% \\
\end{array}$ & $\begin{array}{c}2 \\
20 \% \\
\end{array}$ & $\begin{array}{c}4 \\
40 \% \\
\end{array}$ & \multirow{3}{*}{$\begin{array}{l}\chi^{2}=10.158 \\
P<.01\end{array}$} \\
\hline & 1 & $\begin{array}{c}5 \\
50 \% \\
\end{array}$ & $\begin{array}{c}7 \\
70 \% \\
\end{array}$ & $\begin{array}{c}7 \\
70 \% \\
\end{array}$ & $\begin{array}{c}3 \\
30 \% \\
\end{array}$ & \\
\hline & 2 & $\begin{array}{c}5 \\
50 \% \\
\end{array}$ & $\begin{array}{c}1 \\
10 \% \\
\end{array}$ & $\begin{array}{c}1 \\
10 \% \\
\end{array}$ & $\begin{array}{c}3 \\
30 \% \\
\end{array}$ & \\
\hline \multirow{3}{*}{$\begin{array}{l}\text { Group C } \\
(\mathrm{n}=11)\end{array}$} & $\mathbf{0}$ & $\begin{array}{c}0 \\
0 \%\end{array}$ & $\begin{array}{c}0 \\
0 \%\end{array}$ & $\begin{array}{c}0 \\
0 \%\end{array}$ & $\begin{array}{c}6 \\
54.5 \%\end{array}$ & \multirow{3}{*}{$\begin{array}{l}\chi^{2}=16.524 \\
P<.001\end{array}$} \\
\hline & 1 & $\begin{array}{c}4 \\
36.4 \% \\
\end{array}$ & $\begin{array}{c}8 \\
72.7 \% \\
\end{array}$ & $\begin{array}{c}8 \\
72.7 \% \\
\end{array}$ & $\begin{array}{c}3 \\
27.3 \% \\
\end{array}$ & \\
\hline & 2 & $\begin{array}{c}7 \\
63.6 \% \\
\end{array}$ & $\begin{array}{c}3 \\
27.3 \% \\
\end{array}$ & $\begin{array}{c}3 \\
27.3 \% \\
\end{array}$ & $\begin{array}{c}2 \\
18.2 \% \\
\end{array}$ & \\
\hline $\begin{array}{l}\text { Between the } \\
\text { Group } \\
\text { comparison }\end{array}$ & $\begin{array}{c}\text { PEARSON } \\
\text { Chi-Square } \\
\text { Test }\end{array}$ & $\begin{array}{l}\chi^{2}=0.40 \\
p>.05\end{array}$ & $\begin{array}{l}\chi^{2}=4.46 \\
p>.05\end{array}$ & $\begin{array}{l}\chi^{2}=4.46 \\
p>.05\end{array}$ & $\begin{array}{l}\chi^{2}=1.75 \\
p>.05\end{array}$ & \\
\hline
\end{tabular}

Table 15:-Sandhigati-asamarthya (Restriction of movements)

\begin{tabular}{|l|l|l|c|} 
Group & Grade & No. of cases with percentage & $\begin{array}{c}\text { Within the group } \\
\text { comparison }\end{array}$ \\
\hline
\end{tabular}




\begin{tabular}{|c|c|c|c|c|c|c|}
\hline & & BT & F1 & F2 & F3 & Friedman Test \\
\hline \multirow{4}{*}{$\begin{array}{l}\text { Group A } \\
(n=14)\end{array}$} & $\mathbf{0}$ & $\begin{array}{c}3 \\
21.4 \%\end{array}$ & $\begin{array}{c}3 \\
21.4 \%\end{array}$ & $\begin{array}{c}3 \\
21.4 \%\end{array}$ & $\begin{array}{c}4 \\
28.6 \%\end{array}$ & \multirow{4}{*}{$\begin{array}{l}\chi^{2}=16.867 \\
p<.001\end{array}$} \\
\hline & 1 & $\begin{array}{c}3 \\
21.4 \%\end{array}$ & $\begin{array}{c}3 \\
21.4 \%\end{array}$ & $\begin{array}{c}3 \\
14.3 \%\end{array}$ & $\begin{array}{c}7 \\
50 \% \\
\end{array}$ & \\
\hline & 2 & $\begin{array}{c}5 \\
35.7 \%\end{array}$ & $\begin{array}{c}6 \\
42.9 \%\end{array}$ & $\begin{array}{c}7 \\
50 \%\end{array}$ & $\begin{array}{c}2 \\
14.3 \%\end{array}$ & \\
\hline & 3 & $\begin{array}{c}3 \\
21.4 \% \\
\end{array}$ & $\begin{array}{c}2 \\
14.3 \% \\
\end{array}$ & $\begin{array}{c}1 \\
7.1 \%\end{array}$ & $\begin{array}{c}1 \\
7.1 \%\end{array}$ & \\
\hline \multirow{4}{*}{$\begin{array}{l}\text { Group B } \\
(\mathrm{n}=10)\end{array}$} & $\mathbf{0}$ & $\begin{array}{c}1 \\
10 \%\end{array}$ & $\begin{array}{c}2 \\
20 \%\end{array}$ & $\begin{array}{c}3 \\
30 \%\end{array}$ & $\begin{array}{c}4 \\
40 \%\end{array}$ & \multirow{4}{*}{$\begin{array}{l}\chi^{2}=20.429 \\
p<.001\end{array}$} \\
\hline & 1 & $\begin{array}{c}1 \\
10 \%\end{array}$ & $\begin{array}{c}7 \\
70 \% \\
\end{array}$ & $\begin{array}{c}6 \\
60 \% \\
\end{array}$ & $\begin{array}{c}5 \\
50 \% \\
\end{array}$ & \\
\hline & 2 & $\begin{array}{c}5 \\
50 \%\end{array}$ & $\begin{array}{c}1 \\
10 \%\end{array}$ & $\begin{array}{c}1 \\
10 \%\end{array}$ & $\begin{array}{c}1 \\
10 \%\end{array}$ & \\
\hline & 3 & $\begin{array}{c}3 \\
30 \%\end{array}$ & $\begin{array}{c}0 \\
0 \%\end{array}$ & $\begin{array}{c}0 \\
0 \%\end{array}$ & $\begin{array}{c}0 \\
0 \%\end{array}$ & \\
\hline \multirow{4}{*}{$\begin{array}{l}\text { Group C } \\
(\mathrm{n}=11)\end{array}$} & $\mathbf{0}$ & $\begin{array}{c}0 \\
0 \%\end{array}$ & $\begin{array}{c}1 \\
9.1 \%\end{array}$ & $\begin{array}{c}1 \\
9.1 \%\end{array}$ & $\begin{array}{c}5 \\
45.5 \%\end{array}$ & \multirow{4}{*}{$\begin{array}{l}\chi^{2}=23.964 \\
p<.001\end{array}$} \\
\hline & 1 & $\begin{array}{c}1 \\
9.1 \%\end{array}$ & $\begin{array}{c}4 \\
36.4 \% \\
\end{array}$ & $\begin{array}{c}5 \\
45.5 \% \\
\end{array}$ & $\begin{array}{c}4 \\
36.4 \% \\
\end{array}$ & \\
\hline & 2 & $\begin{array}{c}5 \\
45.5 \%\end{array}$ & $\begin{array}{c}6 \\
54.5 \% \\
\end{array}$ & $\begin{array}{c}5 \\
45.5 \% \\
\end{array}$ & $\begin{array}{c}2 \\
18.2 \%\end{array}$ & \\
\hline & 3 & $\begin{array}{c}5 \\
45.5 \%\end{array}$ & $\begin{array}{c}0 \\
0 \%\end{array}$ & $\begin{array}{c}0 \\
0 \%\end{array}$ & $\begin{array}{c}0 \\
0 \%\end{array}$ & \\
\hline $\begin{array}{l}\text { Between the } \\
\text { Group } \\
\text { comparison }\end{array}$ & $\begin{array}{l}\text { PEARSON Chi- } \\
\text { Square } \\
\text { Test }\end{array}$ & $\begin{array}{l}\chi^{2}=3.916 \\
p>.05\end{array}$ & $\begin{array}{l}\chi^{2}=6.19 \\
p<.05\end{array}$ & $\begin{array}{l}\chi^{2}=5.60 \\
p>.05\end{array}$ & $\begin{array}{l}\chi^{2}=0.55 \\
p>.05\end{array}$ & \\
\hline
\end{tabular}

Table 16:-Womac Score

\begin{tabular}{|c|c|c|c|c|c|}
\hline \multirow{2}{*}{ Groups } & \multicolumn{4}{|c|}{ Mean \pm SD } & \multirow{2}{*}{$\begin{array}{c}\text { Within the group } \\
\text { comparison } \\
\text { Paired t test } \\
\text { BT - AT }\end{array}$} \\
\hline & BT & F1 & F2 & F3 & \\
\hline $\begin{array}{l}\text { Group A } \\
(n=14)\end{array}$ & $\begin{array}{l}40.07 \\
\pm 5.62\end{array}$ & $\begin{array}{c}37.79 \\
\pm 4.60\end{array}$ & $\begin{array}{r}32.29 \\
\pm 3.56\end{array}$ & $\begin{array}{l}27.86 \\
\pm 4.75\end{array}$ & $\begin{array}{l}12.21 \pm 5.31 \\
t=8.61 \\
P<0.001\end{array}$ \\
\hline $\begin{array}{l}\text { Group B } \\
(\mathrm{n}=10)\end{array}$ & $\begin{array}{l}42.30 \\
\pm 5.43\end{array}$ & $\begin{array}{c}28.50 \\
\pm 7.261\end{array}$ & $\begin{array}{r}24.00 \\
\pm 6.02\end{array}$ & $\begin{array}{c}20.60 \\
\pm 5.10\end{array}$ & $\begin{array}{l}21.70 \pm 5.12 \\
t=13.40 \\
P<0.001\end{array}$ \\
\hline $\begin{array}{l}\text { Group C } \\
(\mathrm{n}=11)\end{array}$ & $\begin{array}{l}41.36 \\
\pm 4.88\end{array}$ & $\begin{array}{r}29.73 \\
\pm 5.25\end{array}$ & $\begin{array}{r}24.00 \\
\pm 5.91\end{array}$ & $\begin{array}{c}18.55 \\
\pm 5.46\end{array}$ & $\begin{array}{l}22.82 \pm 4.19 \\
\mathrm{t}=18.06 \\
\mathrm{P}<0.001\end{array}$ \\
\hline $\begin{array}{l}\text { Between the group } \\
\text { comparison } \\
\text { One - Way ANOVA }\end{array}$ & $\begin{array}{l}F=0.52 \\
p>0.05 \\
N S\end{array}$ & $\begin{array}{l}\mathrm{F}=9.92 \\
\mathrm{p}<0.001\end{array}$ & $\begin{array}{l}\mathrm{F}=10.97 \\
\mathrm{p}<0.001\end{array}$ & $\begin{array}{l}F=11.72 \\
p<.001\end{array}$ & - \\
\hline $\begin{array}{ll}\text { Post- Hoc } & \text { Test } \\
\text { Significant pairs } & \\
(\mathrm{p}<0.05) & \\
\end{array}$ & - & $\begin{array}{l}(1,2) \\
(1,3)\end{array}$ & $\begin{array}{l}(1,2) \\
(1,3)\end{array}$ & $\begin{array}{l}(1,2) \\
(1,3)\end{array}$ & - \\
\hline
\end{tabular}


Table 17:-Walking Time

\begin{tabular}{|c|c|c|c|c|c|c|}
\hline \multirow[t]{2}{*}{ Group } & \multirow[t]{2}{*}{ Grade } & \multicolumn{4}{|c|}{ No. of cases with percentage } & \multirow{2}{*}{$\begin{array}{c}\text { Within the group } \\
\text { comparison } \\
\text { Friedman Test }\end{array}$} \\
\hline & & BT & F1 & F2 & F3 & \\
\hline \multirow{4}{*}{$\begin{array}{l}\text { Group A } \\
(n=14)\end{array}$} & $\mathbf{0}$ & $\begin{array}{c}0 \\
0 \%\end{array}$ & $\begin{array}{c}0 \\
0 \%\end{array}$ & $\begin{array}{c}0 \\
0 \%\end{array}$ & $\begin{array}{c}0 \\
0 \%\end{array}$ & \multirow{4}{*}{$\begin{array}{l}\chi^{2}=20.824 \\
p=.001\end{array}$} \\
\hline & 1 & $\begin{array}{c}2 \\
14.3 \%\end{array}$ & $\begin{array}{c}3 \\
21.4 \%\end{array}$ & $\begin{array}{c}6 \\
42.9 \%\end{array}$ & $\begin{array}{c}9 \\
64.3 \% \\
\end{array}$ & \\
\hline & 2 & $\begin{array}{c}7 \\
50 \%\end{array}$ & $\begin{array}{c}7 \\
50 \% \\
\end{array}$ & $\begin{array}{c}5 \\
35.7 \% \\
\end{array}$ & $\begin{array}{c}3 \\
21.4 \% \\
\end{array}$ & \\
\hline & 3 & $\begin{array}{c}5 \\
50 \% \\
\end{array}$ & $\begin{array}{c}4 \\
28.6 \%\end{array}$ & $\begin{array}{c}3 \\
21.4 \% \\
\end{array}$ & $\begin{array}{c}2 \\
14.3 \% \\
\end{array}$ & \\
\hline \multirow{4}{*}{$\begin{array}{l}\text { Group B } \\
(\mathrm{n}=10)\end{array}$} & $\mathbf{0}$ & $\begin{array}{c}0 \\
0 \%\end{array}$ & $\begin{array}{c}4 \\
40 \%\end{array}$ & $\begin{array}{c}4 \\
40 \%\end{array}$ & $\begin{array}{c}3 \\
30 \%\end{array}$ & \multirow{4}{*}{$\begin{array}{l}\chi^{2}=27.403 \\
p<.001\end{array}$} \\
\hline & 1 & $\begin{array}{c}2 \\
20 \%\end{array}$ & $\begin{array}{c}3 \\
30 \%\end{array}$ & $\begin{array}{c}4 \\
40 \%\end{array}$ & $\begin{array}{c}5 \\
50 \%\end{array}$ & \\
\hline & 2 & $\begin{array}{c}4 \\
40 \% \\
\end{array}$ & $\begin{array}{c}3 \\
30 \% \\
\end{array}$ & $\begin{array}{c}2 \\
20 \% \\
\end{array}$ & $\begin{array}{c}2 \\
20 \% \\
\end{array}$ & \\
\hline & 3 & $\begin{array}{c}4 \\
40 \% \\
\end{array}$ & $\begin{array}{c}0 \\
0 \%\end{array}$ & $\begin{array}{c}0 \\
0 \% \\
\end{array}$ & $\begin{array}{c}0 \\
0 \%\end{array}$ & \\
\hline \multirow{4}{*}{$\begin{array}{l}\text { Group C } \\
(n=11)\end{array}$} & $\mathbf{0}$ & $\begin{array}{c}0 \\
0 \% \\
\end{array}$ & $\begin{array}{c}0 \\
0 \% \\
\end{array}$ & $\begin{array}{c}1 \\
9.1 \% \\
\end{array}$ & $\begin{array}{c}4 \\
36.4 \% \\
\end{array}$ & \multirow{4}{*}{$\begin{array}{l}\chi^{2}=23.081 \\
\mathrm{p}<.001\end{array}$} \\
\hline & 1 & $\begin{array}{c}1 \\
9.1 \%\end{array}$ & $\begin{array}{c}5 \\
45.5 \%\end{array}$ & $\begin{array}{c}5 \\
45.5 \%\end{array}$ & $\begin{array}{c}3 \\
27.3 \% \\
\end{array}$ & \\
\hline & 2 & $\begin{array}{c}5 \\
45.5 \% \\
\end{array}$ & $\begin{array}{c}5 \\
45.5 \% \\
\end{array}$ & $\begin{array}{c}4 \\
36.4 \% \\
\end{array}$ & $\begin{array}{c}3 \\
27.3 \% \\
\end{array}$ & \\
\hline & 3 & $\begin{array}{c}5 \\
45.5 \%\end{array}$ & $\begin{array}{c}1 \\
9.1 \%\end{array}$ & $\begin{array}{c}1 \\
9.1 \%\end{array}$ & $\begin{array}{c}1 \\
9.1 \%\end{array}$ & \\
\hline $\begin{array}{l}\text { Between the } \\
\text { Group } \\
\text { comparison }\end{array}$ & $\begin{array}{c}\text { PEARSON } \\
\text { Chi-Square } \\
\text { Test }\end{array}$ & $\begin{array}{l}\chi^{2}=0.51 \\
p>.05\end{array}$ & $\begin{array}{l}\chi^{2}=5.66 \\
p>.05\end{array}$ & $\begin{array}{l}\chi^{2}=3.33 \\
p>.05\end{array}$ & $\begin{array}{l}\chi^{2}=0.85 \\
p>.05\end{array}$ & \\
\hline
\end{tabular}

Table 18:-Showing the distribution of Overall Response to the treatment:

\begin{tabular}{|l|c|c|c|}
\hline \multicolumn{1}{|c|}{ Response } & $\begin{array}{c}\text { Group A } \\
\text { No. and percentage }\end{array}$ & $\begin{array}{c}\text { Group B } \\
\text { No. and percentage }\end{array}$ & $\begin{array}{c}\text { Group C } \\
\text { Nond percentage }\end{array}$ \\
\hline Good Response & 5 & 4 & 7 \\
& $35.7 \%$ & $40 \%$ & $63.6 \%$ \\
\hline Moderate Response & 7 & 5 & 3 \\
& $50 \%$ & $50 \%$ & $27.3 \%$ \\
\hline Poor Response & 2 & 1 & 1 \\
& $14.3 \%$ & $10 \%$ & $9.1 \%$ \\
\hline No Response & 0 & 0 & 0 \\
& $0 \%$ & $0 \%$ & $0 \%$ \\
\hline
\end{tabular}

\section{Discussion:-}

In the present study, maximum number of patients were of age Group 41-50 years (46.66\%) followed by 51-60 years age Group (26.66\%). Sandhigatavata is the disease of Vriddhavastha due to Dhatukshaya which has been found same here in the present study. Majority of the patients were of Female sex $(62.22 \%)$ in the present study. Male sex found in $37.77 \%$ patients. In this study $(55.55 \%)$ patients were doing active occupation. Due to the continuous stress affecting on the joints due to excessive work the particular joint becomes degenerated causing Sandhigatavata (Osteoarthritis). (31.11\%) patients were of sedentary. Life style and occupation are regarded as the 
risk factors in the epidemiology of Osteoarthritis in contemporary medical science. (Arthritis clinical symposia.vol.51.1999).

Maximum number of patients were having chronicity of 1-2 years (33.33\%), followed by chronicity of more than 5 years $(22.22 \%)$ and 2-5 years of Chronicity (17.77\%).The disease Sandhigatavata is encountered as a Yapya or Kastasadhya variety of disease under the Vatavyadhi and all the Yapyavikaras are chronic in nature. As per observations, the chronicity of the Sandhigatavata is reflected. The chronicity is inversely proportional to the prognosis of disease i.e. if chronicity is less, prognosis is good. In this series, $80 \%$ patients gave history of Gradual onset while $20 \%$ patients gave history of Insidious onset of the disease. Gradual slow progression of the disease Osteoarthritis is common. Data of the present study shows the same thing.

\section{Therapeutic response of the clinical trial :}

Statistically highly significant relief was obtained in all the groups in the complain of prasaran-akunchanyohvedana ( Pain ) as $\mathrm{p}<.001$ in all the groups but percentage relief in pain was more in Group C than group B, and group B more than Group A. Statistically significant result was obtained in between the group comparison after the treatment as $\mathrm{p}<.05$.

Statistically not significant relief was obtained in Group A in complain of Stambha (Stiffness) as p > .05. In Group $\mathrm{B}$ and $\mathrm{C}$ highly significant result was obtained $(\mathrm{p}<0.001)$. Degree of improvement was more in group $\mathrm{C}$ than group B. Highly significant result was obtained on between the groups comparison as $\mathrm{p}<.05$.

All the groups have highly significant relief in complain of Sparshakshamatwa (tenderness) as $\mathrm{p}<.001$ in all the groups. Between the groups comparison result was insignificant, percentage relief was highest in group C, and B have more percentage relief than group A.

Similarly all the groups have highly significant improvement in Shotha (swelling), Sandhigati-asamarthya (restriction of movement), Atopa (crepitus) and Walking time as $\mathrm{p}<.001$ in all the groups in all the symptoms. Between the Groups comparison was not significant. Degree of improvement by seeing the percentage relief, effect was highest in group C than Group B, group B more effective than group A.

WOMAC Score was significantly reduced in all the groups after trial therapy as $\mathrm{p}$ is $<.001$ in all the groups. The mean difference was $12.21 \pm 5.31$ in Group A. While it was $21.70 \pm 5.12$ in Group B and $22.82 \pm 4.19$ in group C. On intergroup comparison statistical significance was obtained in WOMAC score between Group A and B and Group A and C.

In Group A, B and C, 35.7\%, 40\% and 63.6\% patient respectively had the good response, 50\%, 50\% and $27.3 \%$ have moderate response respectively and $14.3 \%, 10 \%$ and $9.1 \%$ respectively shows poor response. Any group didn't have No response in any patient.

Shamana therapy as Rasnadi Guggulu provided also better result in all signs and symptoms but low in percentage in comparison to Basti (shodhana) and Basti (Shodhana) followed by administration of Rasnadi Guggulu (shaman).

In this study the Basti therapy has shown beneficial effects on subjective and objective criteria of the patients of Sandhigatavata.

\section{Probable Mode Of Action \\ Rasnadi Guggulu}

The Rasnadi Guggulu can reduce the symptoms as well as the degenerative process due to various actions of individual drugs in it. The contents of this composition are all Vata Shamaka. The drugs like Rasna, Eranda, Devadaru are not only capable of pacifying Vata but also is very good in promoting the health by normalising the Doshas. This composition can also help to control the progressive degeneration, desquamation and necrosis of perichondreal tissues and chondrocytes of the articular cartilage. Rasna is a known anti-inflammatory drug as well as disease modifying agents. Amrita, Shunthi, Devadaru have immunomodulatory action from which can regulate the 
lysosomal secretion macrophages in the synovial fluids. Therefore this Rasnadi Guggulu provides symptomatic relief from inflammation and arthralgia through its anti-inflammatory, analgesic and possibly anti kinin effects.

\section{Panchamulabaladi Ksheer Basti}

Brihatpanchmula is having well known anti-inflammatory and analgesic action. Brihatpanchmula is the most highly prized Ayurvedic formula for fatigue. It aids cellular regeneration to hasten removal of dead or weak cells and replace them with fresh ones. Bala acts on nerves and is Balya, \& Vatahara, it is also Brimhan \& Ojovardhaka. Bala is a drug of choice amongst all Balya and Vatahara ${ }^{26}$ drugs. Balamula has Rasayana and Ojovardhaka properties. Acharya Dalhana has considered Purishdhara kala as Asthidhara kala ${ }^{27}$. Purishdhara kala is located in Pakwashaya. It is very important site of vata dosha ${ }^{28}$. The virya of Bastidravya, because of its Anupravanabhava, is transmitted to all over the body by the Apanadi vatas and thus has a systemic effect. The virya of the bastidravya extracts the morbid doshas from all parts of the body i.e. "ApadaMastaka" to the pakwashaya ${ }^{28}$. By the combined effect of purvakarma viz Amapachana, Snehana and Swedana, the morbid doshas are brought to kostha. The Pharmacodynamics of these three procedures is nothing but Paka, Vishyandana, Srotomukha vishodhana and Vayunigrahana ${ }^{29}$. These factors are responsible for the movement (Gati) of Doshas from Shakha to Kostha. The overall action of Dravyas of Panchmulabaladi Ksheer Basti, the prominent rasa is madhura and madhura vipaka which are Saptadhatuvardhak, Balya, Sthairyakara, Kshatasandhanakara and vata-kaphashamak and Rasayana properties ${ }^{30}$. Tikta rasa following is lekhana, kleda-meda-kapha soshana, Dipana and Pachana ${ }^{31}$. Tikta Ksheer basti are especially indicated in diseases of Asthi dhatu ${ }^{32}$. Katu rasa is shotha, kleda nashana, lekhana and Margavivarana ${ }^{33}$. Kashayarasa is Sandhanakara, Ropana, Shosana, Lavana rasa is in trace, it is margavishodhana, Stambha, Bandha, Sanghatakara. Looking at virya bastidravya is prominently usna virya which does Swedana, Pachana, Vilayana, and Vata-kapha nashana ${ }^{34}$. On analysing effect on Tridosha, it is mainly vataghna, kaphaghna and pittaghna also. It indicates Tridoshaghnata of Bastidravya. When studying gunas, Snigdha and Guru gunas are prominent. Snigdhaguna is malapravartak. It produces snigdhata (oleation), mriduta (softness) and ardrata (Moisturisation) in the body. It is vatahara and Balya ${ }^{35}$. Guru guna has Adhogamipravritti (Towards gravity) which helps in elimination of malas. It is Dhatuvardhak, Balya, Vatahara as well ${ }^{36}$. Laghu follows guru guna which is lekhana, Ropana, Kaphaghna ${ }^{37 .}$ Ruksha is soshana, kaphanashaka. Tiksna and usna gunas are shodhana, lekhana and kaphavatahara. Sukshma guna of basti drugs has the power to penetrate the deepest and smallest srotas of the body. Pichchila is tantula and styana hence it helps in sandhana karma ${ }^{38}$ of the organs. Overall effect of Bastidravya is mainly shoolaghna, sothaghna and Balya. In addition to these prominent karmas, bastidravya may have yogavahi lakshana. Tridoshahara property because of Madhu and due to Saindhav, dipana pachana karma is made. Rasayana properties of ksheer and bala helps in the rejuvenation of the tissues.

\section{Conclusion:-}

The Sandhigatavata explained in Ayurvedic classics as Sthana vishesha krita Vatavyadhi, under the concept of Gatavata. The vitiated Vata dosha get lodged in Asthi- Sandhis and give rise to Sandhigatavata. This is one of such disease commonly affecting a large number of elderly individuals. Rasnadi Guggulu has properties like Vedanasthapana, Shothahara, Balya, Rasayana, Sandhaniya, Deepana, Anulomana etc. Its pharmacological activities include anti- inflammatory, analgesics, antioxidant, immunostimulant and rejuvenator, by these properties the drug is beneficial for shaman of Sandhigatavata. Basti is best known treatment for vatavyadhi, amongst Basti, ksheer. The disease Sandhivata is mainly due to Dhatukshaya and Bala kshaya. The ingredients of the given Basti eg Brihatpanchmula is Vatashamak, Shothahara and Balya. Bala used in the Basti provides to the body having Vatashamana and Rasayana properties. So it is very good in Vatavyadhi i.e. in degenerative disorders. Its pharmacological activities include Rejuvenative, Anti inflammatory, Analgesic etc. Goksheer present in the basti is jeevaniya, rasayana, balya, dhatuposhaka and vrishya. The trial drug showed excellent response on reduction of the symptoms and in degenerative process. It is well observed that shodhana group showed better result than the shamana group. It is well assessed that shodhana should carried out before commencing internal medication. The size of sample was small to draw a generalized conclusion. The period of study was limited. The study can be repeated with large sample and longer duration.

\section{Reference:-}

1. Vaidya Ranajeet Rai Desai, Ayurvediya kriya sharir, Sri Baidyanath Ayurveda Bhawan Limited,Naini, Allahabad, $4^{\text {th }}$ edition 2006, page 9. 
2. Osteoarthritis and ageing- Ana M. Valdes, Jo anne stocks, European medical journal, March 2018-Page-116121, https://www.emjreviews.com/rheumatology/article/osteoarthritis-and-ageing.

3. Osteoarthritis and ageing- Ana M. Valdes, Jo anne stocks, European medical journal, March 2018-Page-116121, https://www.emjreviews.com/rheumatology/article/osteoarthritis-and-ageing.

4. Vagbhata, Ashtanga Hridayam, Saarvangasundara commentary by Arunadatta and Vidyotini commentary by Atrideva Gupta, edited by Prof. P.V.Sharma, Reprint edition 2009, Sutra sthana, chapter 1/7,page 7, Chaukhamba Orientalia, Varanasi.

5. Agnivesha ,Charaka Samhita, elaborated by Charak and Drdhbala, edited and translated by Prof. P.V.Sharma, volume $1,9^{\text {th }}$ edition 2004, Chaukhambha Orientalia, Varanasi, Vimana sthana, chapter 8/122, page 383.

6. Agnivesha, Charaka Samhita , refined and annotated by Charaka and redacted by Drdhbala, edited and translated by Prof. P.V. Sharma, Vol.2, $7^{\text {th }}$ edition, 2005, Chaukhambha orientalia, Varanasi. Chikitsa Sthana, 28/18, Page- 462

7. Vagbhata, Ashtanga Hridayam, Saarvangasundara commentary by Arunadatta and Vidyotini commentary by Atrideva Gupta, edited by Prof. P.V.Sharma, Reprint edition 2009, Chaukhambha orientalia, Varanasi. Sutra sthana, chapter $12 / 17$, page 158 .

8. Agnivesha ,Charaka Samhita, elaborated by Charaka and Drdhbala, edited and translated by Prof. P.V.Sharma, volume $1,9^{\text {th }}$ edition 2005, Chaukhambha Orientalia, Varanasi, Vimana sthana, chapter 8/122, page 383

9. Agnivesha ,Charaka Samhita, elaborated by Charaka and Drdhbala, edited and translated by Prof. P.V.Sharma, volume $1,9^{\text {th }}$ edition 2004, Chaukhambha Orientalia, Varanasi, Sutra sthana, chapter 11/48, page 78.

10. Agnivesha ,Charaka Samhita, elaborated by Charaka and Drdhbala, edited and translated by Prof. P.V.Sharma, volume 1, $9^{\text {th }}$ edition 2004, Chaukhambha Orientalia, Varanasi, Sutra sthana, chapter 10/18, page 68.

11. Chandra Prakash Pal, Pulkesh singh, Sanjay chaturvedi et al, Epidemiology of knee osteoarthritis in India and related factors, Indian journal of orthopaedics. 2016 Sep, page 518-522.

12. Chandra Prakash Pal, Pulkesh singh, Sanjay chaturvedi et al, Epidemiology of knee osteoarthritis in India and related factors, Indian journal of orthopaedics. 2016 Sep, page 518-522.

13. Chandra Prakash Pal, Pulkesh singh, Sanjay chaturvedi et al, Epidemiology of knee osteoarthritis in India and related factors, Indian journal of orthopaedics. 2016 Sep, page 518-522.

14. Pandelton A, Arden N, Dougados M, et al. EULAR recommendations for the management of knee osteoarthritis: report of a task force of the Standing Committee for International Clinical Studies Including Therapeutic Trials (ESCISIT), Ann Rheum Dis 2000;59: 936-44.

15. David J Hunter, BMJ- 2006 Mar 18, 332(7542) page- 639-642, doi: 10.1136/ bmj.332.7542.639. PMCID: PMC 1403209 PMID: 16543327. www.ncbi.nlm.nih.gov/pmc/articles/pmc

16. Yogaratnakara, Vaidyak Granth, edited by Pt. Sadasiva Sastri Joshi, $2^{\text {nd }}$ edition 2007, Chaukhamba Sanskrit Series Office Varanasi, chapter - Vatavyadhi nidanam, page 413.

17. Bhaisajya Ratnavali of Kaviraj Govind Das Sen, edited with Siddhiprada Hindi commentary by Prof. Siddhi Nandan Mishra, Chaukhamba Subharati Prakashan, Varanasi, Reprint edition -2009, chapter 26/5, page 518.

18. Cakradatta of Shri Cakrapani data, Edited and translated by Dr.P.V.Sharma, Edition 2007, Chaukhambha Orientalia, Varanasi, Vatavyadhi Adhikara 22/83, page 193.

19. Agnivesha, Charaka Samhita , refined and annotated by Charaka and redacted by Drdhbala, edited and translated by Prof. P.V. Sharma, Vol.2, $7^{\text {th }}$ edition, 2005, Chaukhambha orientalia, Varanasi, Siddhi Sthana, chapter 1/47-48, page 594 .

20. Sushruta, Sushruta Samhita, with Dalhana Commentary, volume 2, edited and translated by Prof. P.V.Sharma, Chaukhambha Orientalia, Varanasi, Reprint 2010, Nidana Sthana, chapter 1/28.

21. Sushruta, Sushruta Samhita, with Dalhana Commentary, volume 2, edited and translated by Prof. P.V.Sharma, Chaukhambha Orientalia, Varanasi, Reprint 2010, Nidana Sthana, chapter 1/28.

22. Agnivesha, Charaka Samhita , refined and annotated by Charaka and redacted by Drdhbala, edited and translated by Prof. P.V. Sharma, Vol.2, $7^{\text {th }}$ edition, 2005, Chaukhambha orientalia, Varanasi, Chikitsa sthana, chapter 28/37, page- 463 .

23. Madhava Nidanam, Roga Vinischaya of Sri Madhavakara with the sanskrit commentary Madhukosha by Vijayarakshita and Srikanthadatta, edited with 'Vimala', 'Madhudhara' Hindi commentary and notes by Dr. Brahmananda Tripathi, Volume 1, Chaukhamba subharati Prakashana, Varanasi, chapter 22/21, page 520. 
24. Agnivesha, Charaka Samhita , refined and annotated by Charaka and redacted by Drdhbala, edited and translated by Prof. P.V. Sharma, Vol.2, $7^{\text {th }}$ edition, 2005, Chaukhambha orientalia, Varanasi, Chikitsa sthana, chapter 28/37, page- 463 .

25. http://www.rheumatology.org/practice/clinical/clinicianresearchers/outcomes-instrumentation/WOMAC.asp.

26. Agnivesha ,Charaka Samhita, elaborated by Charak and Drdhbala, edited and translated by Prof. P.V.Sharma, volume $1,9^{\text {th }}$ edition 2004, Chaukhambha Orientalia, Varanasi, Sutra Sthana, chapter 25/40, page 168.

27. Sushruta, Susruta Samhita with the Nibandhasangraha Commentary of Sri Dalhanacharya and the Nyayachandrika Panjika of Sri Gayadasacharya on Nidanasthana, edited by Vaidya Jadavji Trikamji Acharya and Narayan Ram Acharya, Chaukhamba Subharati Prakashan Varanasi, Reprint 2003, Kalpa sthana, Chapter 4/40,Page- 574.

28. Agnivesha, Charaka Samhita , refined and annotated by Charaka and redacted by Drdhbala, edited and translated by Prof. P.V. Sharma, Vol.2,7 ${ }^{\text {th }}$ edition, 2005, Chaukhambha orientalia, Varanasi, Siddhi Sthana, chapter 1/40, page-592.

29. Agnivesha ,Charaka Samhita, elaborated by Charak and Drdhbala, edited and translated by Prof. P.V.Sharma, volume $1,9^{\text {th }}$ edition 2004, Chaukhambha Orientalia, Varanasi, Sutra sthana, chapter 28/33, page 230.

30. Agnivesha ,Charaka Samhita, elaborated by Charak and Drdhbala, edited and translated by Prof. P.V.Sharma, volume $1,9^{\text {th }}$ edition 2004, Chaukhambha Orientalia, Varanasi, Sutra sthana, chapter 26/43(1), page 181.

31. Agnivesha ,Charaka Samhita, elaborated by Charak and Drdhbala, edited and translated by Prof. P.V.Sharma, volume $1,9^{\text {th }}$ edition 2004, Chaukhambha Orientalia, Varanasi, Sutra sthana, chapter 26/43(5), page 182.

32. Agnivesha ,Charaka Samhita, elaborated by Charak and Drdhbala, edited and translated by Prof. P.V.Sharma, volume $1,9^{\text {th }}$ edition 2004, Chaukhambha Orientalia, Varanasi, Sutra sthana, chapter 28/27, page 230.

33. Agnivesha ,Charaka Samhita, elaborated by Charak and Drdhbala, edited and translated by Prof. P.V.Sharma, volume 1, $9^{\text {th }}$ edition 2004, Chaukhambha Orientalia, Varanasi, Sutra sthana, chapter 26/43(4), page 182.

34. Vagbhata, Astanga Samgraha, volume 1, Sutra Sthana, translated by Prof. K.R.Srikantha Murthy, Chaukhambha Orientalia, Varanasi, $2^{\text {nd }}$ edition, chapter $17 / 22$, page -325.

35. Susruta, Susruta Samhita, with Dalhana commentary, volume 1, edited and translated by Priya Vrat Sharma, Chaukhambha Visvabharati, reprint 2010, Sutra sthana, chapter 46/516, page 559.

36. Susruta, Susruta Samhita, with Dalhana commentary, volume 1, edited and translated by Priya Vrat Sharma, Chaukhambha Visvabharati, reprint 2010, Sutra sthana, chapter 46/518, page 559.

37. Susruta, Susruta Samhita, with Dalhana commentary, volume 1, edited and translated by Priya Vrat Sharma, Chaukhambha Visvabharati, reprint 2010, Sutra sthana, chapter 46/519, page 559.

38. Susruta, Susruta Samhita, with Dalhana commentary, volume 1, edited and translated by Priya Vrat Sharma, Chaukhambha Visvabharati, reprint 2010, Sutra sthana, chapter 46/517, page 559. 